\title{
Click Through Rate Utilization Approches and its Limitation: A Review
}

\author{
Shraddha Sharma \\ Shri Shankaracharya Technical Campus \\ Dept. of Computer Science and Engineering \\ Bhilai, Chhattisgarh, India
}

\author{
Abhishek Kumar Dewangan \\ Asst Prof. \\ Shri Shankaracharya Technical Campus \\ Dept. of Computer Science and Engineering \\ Bhilai, Chhattisgarh, India
}

In today's Internet world each one experiences the web straight forwardly or in a roundabout way. Current programming based frameworks gather data about their activity and history in click through file. These files data can be used for analysis. The click through document having timestamps, IP address, month, date, and so forth. In today's web situation click through record examination get to be distinctly vital undertaking for dissecting the client's conduct and for enhancing the web applications and managing an account frameworks, and so forth. Rapid change in the technology, make analysis of these file to predict how system will behave in an uncertain situations. Hadoop and apache spark are well known parallel processing system. HDFS and MapReduce are parallel processing systems. Click through files which are created by the web servers contain information about the activities of the visitors like number of visitors and from which domain they are visiting. Thus analyzing those file are very tedious tasks. This paper present some of the techniques used to analyses Hit count of the users and present appropriate method for processing in parallel environment using click through document.

\section{Keywords}

Hadoop, HDFS, Hit Count, Click Throgh Document.

\section{INTRODUCTION}

In today's extending world, everything is going on the web. Sectors like private, public and business has seen a noteworthy improvement in their particular fields. There has been an exponential development in information over the web. We have to dissect these information to give better support of different parts to enhance venture situation. Information is heterogeneous in nature and investigation of such information will give us essential data wherein log documents give an effective arrangement. Log documents are situated in the web server and it contains data about each individual's solicitations, which is put away in a log passage. The fundamental reason for utilizing Hadoop MapReduce is to investigate the datasets successfully.

Click Through documents are produced ordinary which are in the request of terabytes. Click through documents contain colossal measures of valuable data which can helpful to enhance business endeavors and future appraisal. Keeping in mind the end goal to pick up information about the client's exercises, regardless of whether he is buying the item, in the event that he is finding the application cordial to utilize or the issues he is confronting and how it can be settled, we have to investigate click through records. In this manner through click through document investigation, we pick up knowledge into all the previously mentioned inquiries and association of individuals with web applications.

Formula of Click Through rate given by:

$$
C T R=\frac{\text { Clicks }}{\text { Impressions }} * 100
$$ \\ CTR - Click through Rate \\ Clicks - users who click on the link.
}

Impressions - number of people who viewed link.

\subsection{Apache Hadoop and its Architecture}

The Apache Hadoop programming library is a system that takes into account the distributed processing of extensive information sets crosswise over clusters of PCs utilizing simple programming models. It is designed to scale up from single servers to a large number of machines, every offering neighborhood computation and storage.

As opposed to depend on equipment to convey highaccessibility, the library itself is intended to recognize and handle failures at the application layer, so conveying an exceedingly accessible administration on top of a cluster of PCs, each of which might be inclined to failure.

\subsection{MapReduce Architecture}

Hadoop MapReduce is a software framework for executing tremendous measure of information i.e. terabyte data sets in parallel environment on large clusters (in a huge number of data nodes) which can be commodity hardware in a fault tolerant manner.

MapReduce jobs splits the input information set into different pieces of files which then are handled by the guide assignments in parallel form. The hadoop framework sorts the output of map phase which are then input to the reduce tasks. Both input and output files are stored on HDFS (Hadoop Distributed File System). The Hadoop framework has a duty of managing and scheduling tasks.

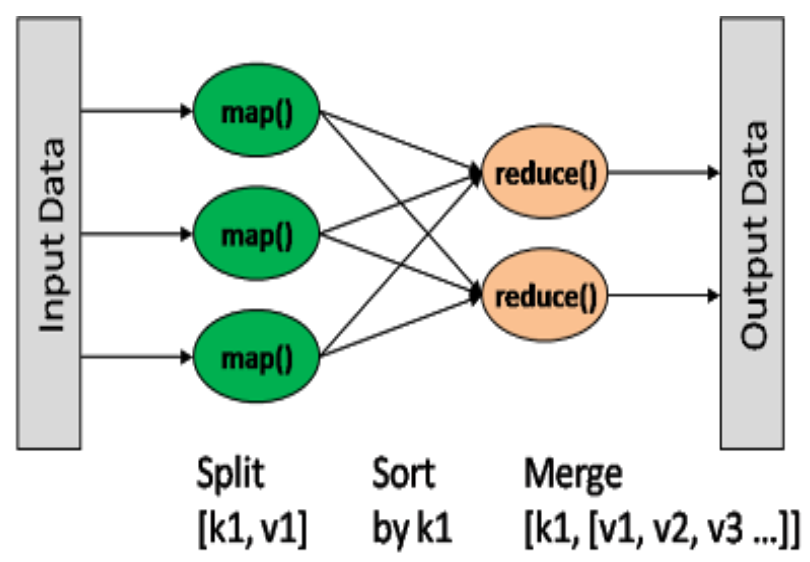

Fig. 1. Shows workflow of MapReduce 


\section{LITERATURE SURVEY}

Lili Shan et al. [1] compare the performance and runtime complexity of our method with Tucker decomposition, canonical decomposition and other popular methods for CTR prediction over real-world advertising datasets. Our experimental results demonstrate that the improved model not only achieves better prediction quality than the others due to considering fully coupled interactions between three entities, user, publisher and advertiser but also can accomplish training and prediction with linear runtime.

Sayalee Narkhede et al. [2] applied this Hadoop MapReduce programming model for analyzing web log files so that we could get hit count of specific web application. This system uses Hadoop file system to store log file and results are evaluated using Map and Reduce function. Experimental results show hit count for each field in log file. Also due to MapReduce runtime parallelization response time is reduced.

Marlina Abdul Latib et al. [3] highlight the characteristics of Big Data as well as Hadoop Framework that has been widely used as Big Data application. Results from the papers reviewed shows that majority researchers applied MapReduce as the main component of Hadoop for analyzing the log files and HDFS as the data storage. Previous researchers have also used other tools and algorithms together with the Hadoop Framework for analyses purposes. The findings of this paper provide a comprehensible review of Hadoop usage performance in analyzing different types of $\log$ files and recommend understandable results for end users to use in future work

Avneesh Tiwari et al., [4] analyses huge datasets need a parallel processing mechanism. Hadoop and apache spark are well known parallel processing system. HDFS and MapReduce are parallel processing systems. In this paper, authors propose a log analysis system which is run over hadoop MapReduce and Apache Spark environment. Both the framework proposes log data in parallel system using all the mechanism in the hadoop and spark cluster and computes result efficiently. Output in the form of some parameters of log such as IP addresses, month, date, time etc. so that it can be useful for real time projects, companies, banks etc.

Xuerui Wang et al. [5] develops models and methods to smoothen CTR estimation by taking advantage of the data hierarchy in nature or by clustering and data continuity in time to leverage information from data close to the events of interest. In a contextual advertising system running at Yahoo!, we demonstrate that our methods lead to significantly more accurate estimation of CTRs.

Table 1. Shows Comparison between Various Existing Approaches and its Limitation

$\begin{array}{cccc}\text { Ref... } & \text { Dataset Used } & \begin{array}{c}\text { Application } \\ \text { Area }\end{array} & \text { Algorithms } \\ & & & \\ & & & \\ \text { Lili Shan et } & \text { Publisher } & \text { Tracking } & \text { Advertisemen } \\ \text { al. } & \text { Dataset } & \text { t } & \text { Factorization }\end{array}$

$\begin{array}{cccc}\text { Sayalee } & \text { Web Logs of } & \text { Tracking } & \text { MapReduce / } \\ \text { Narkhede et } & \begin{array}{c}\text { user Click } \\ \text { Logs }\end{array} & \text { Cloud }\end{array}$

\begin{tabular}{|c|c|c|c|}
\hline $\begin{array}{l}\text { Marlina } \\
\text { Abdul Latib } \\
\text { et al. }\end{array}$ & $\begin{array}{c}\text { Web Server } \\
\text { Dataset }\end{array}$ & $\begin{array}{c}\text { Tracking } \\
\text { Logs }\end{array}$ & MapReduce \\
\hline Avneesh & Web Log & $\begin{array}{l}\text { Counting } \\
\text { User Rate }\end{array}$ & Hadoop / Spark \\
\hline
\end{tabular}

Tiwari User Rate

Xuerui

Wang
Yahoo! Web

$\begin{array}{cc}\text { Yahoo Advert } & \text { Yahoo! Web } \\ \text { Dataset } & \text { Advertisemen } \\ \mathrm{t}\end{array}$

Empirical Bayes Method

\section{Approach Used}

Tucker decomposition, canonical

decomposition and other popular methods for CTR prediction over real-world advertising datasets.

This system uses Hadoop file system to store log file and results are evaluated using Map and Reduce function.

Utilized Hadoop MapReduce Framework in pseudo distributed mode.

Processes data in parallel system using all the mechanism in the hadoop and spark cluster and computes result efficiently.

Smoothen CTR estimation by taking advantage of the data hierarchy in nature or by clustering and data continuity in time to leverage information from data close to the events of interest.

\section{Limitations}

Uses Standalone System

Size of dataset is taken small hence need for improvement.

Works in pseudo distributed mode only.

Response time is
more

Only 60\% Deduction in average KL divergence against the baseline without smoothing. 


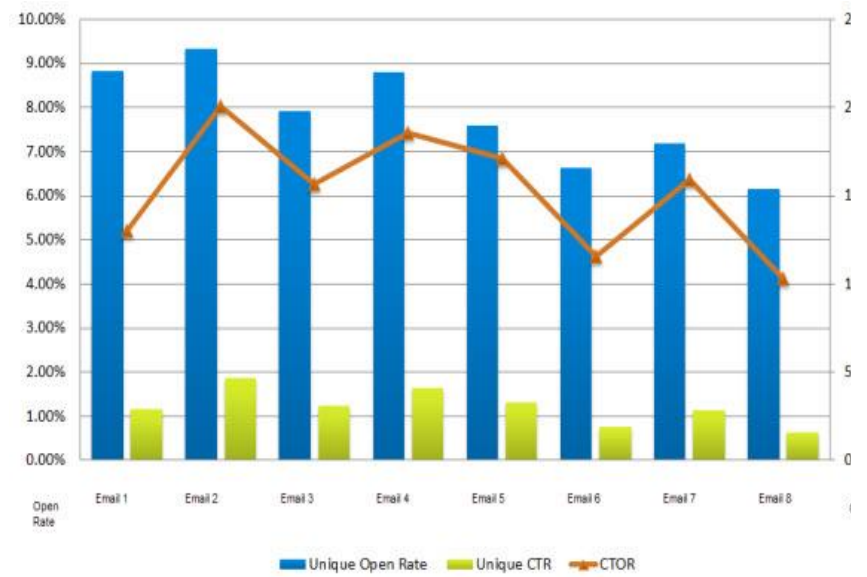

Fig.2. Shows Click through rate of Email Links

\section{CONCLUSION}

Evaluating CTRs for uncommon occasions are critical however exceptionally difficult. We outlined various strategies to manage the sparsely issue lying in the uncommon occasions. The strategy such as Bayes based empirical formula or Map Reduce Framework based approach, they both are succeeded in process the huge file. But have some disadvantages such as slow processing time, higher response time etc. CTR has to be analyzed in very small duration of time due to target many number of users effectively. Numerous web based promoting conditions depends on Click through rates.

\section{REFERENCES}

[1] Lili Shan, Lei Lin, Chengjie Sun, Xiaolong Wang, "Predicting ad click-through rates via feature-based fully coupled interaction tensor factorization", Journal Electronic Commerce Research and Applications archive, Volume 16 Issue C, March 2016, Pages 30-42, ISSN: 1567-4223

[2] Narkhede, S., T. Baraskar, and D. Mukhopadhyay. 2014. Analyzing Web Application Log Files to Find Hit Count through the Utilization of Hadoop MapReduce in Cloud Computing Environment. In 2014 Conference on IT in Business, Industry and Government (CSIBIG),IEEE, MARCH 2015, Page 1-7.

[3] Marlina Abdul Latib, Saiful Adli Ismail, Haslina Md Sarkan and Rasimah Che Mohd Yusoff , "ANALYZING LOG IN BIG DATA ENVIRONMENT", ARPN Journal of Engineering and Applied Sciences ,VOL. 10, NO. 23, DECEMBER 2015 ISSN 1819-6608.

[4] Avneesh Tiwari, Rishabh Soni, Dr. Sanjay Agrawal ,"WEB LOG MINING USING MAPREDUCE AND APACHE SPARK", International Journal of Engineering ResearchOnline, Vol.3., Issue.5., 2015 (Sept.-Oct.).

[5] Zhipeng Fang, Kun Yue, Jixian Zhang, Dehai Zhang, and Weiyi Liu, "Predicting Click-Through Rates of New Advertisements Based on the Bayesian Network," Mathematical Problems in Engineering, vol. 2014, Article ID 818203, 9 pages, 2014. doi:10.1155/2014/818203. 